\title{
Análise cinemática da marcha em indivíduos com hemiparesia espástica após acidente vascular cerebral
}

\author{
Kinematic analysis of gait in individuals with spastic hemiparesis secondary to stroke
}

\author{
Patrick Jacobsen Westphal', Jussara Ferreira' ${ }^{1}$, Vinícius Mazzochi Schimit ${ }^{2}$, Fernanda Cecheti ${ }^{3}$, \\ Leandro Viçosa Bonetti ${ }^{4}$, Raquel Saccani ${ }^{4} \bowtie$ \\ ${ }^{1}$ Centro de Ciências Biológicas e da Saúde, Universidade de Caxias do Sul (UCS). Caxias do Sul, RS \\ 2 Instituto de Medicina do Esporte e Ciências Aplicadas ao Movimento Humano, Laboratório de Análises Biomecânicas do Movimento Humano da UCS. \\ Caxias do Sul, RS. \\ ${ }^{3}$ Universidade Federal de Ciências da Saúde de Porto Alegre. Porto Alegre, RS. \\ ${ }^{4}$ Curso de Fisioterapia da UCS. Caxias do Sul, RS.
}

\section{RESUMO}

Objetivos: Avaliar a cinemática linear e angular da marcha em pacientes hemiparéticos espásticos após acidente vascular cerebral, considerando os valores da normalidade e o membro não afetado.

Métodos: Estudo transversal, no qual participaram indivíduos adultos com hemiparesia espástica após acidente vascular cerebral, selecionados na Clínica de Fisioterapia da Universidade de Caxias do Sul. A análise cinemática da marcha foi realizada no Laboratório de Análises Biomecânicas do Movimento Humano da mesma instituição, seguindo o protocolo de Laroche. Para análise de dados foi utilizada estatística descritiva, teste $t$ para média de uma amostra e teste $t$ pareado. O nível de significância adotado foi $\mathrm{p}<0,05$.

Resultados: Foram estudados oito indivíduos adultos, com idade entre 43 a 59 anos, de ambos os sexos. Foi identificada diferença significativa na cinemática linear da marcha do membro afetado para todas as variáveis estudadas. Considerando a comparação entre os membros inferiores, o comprimento do passo e o tempo do passo foram significativamente maiores no hemicorpo acometido. Com relação às variáveis angulares, os indivíduos demonstraram diferença significativa da normalidade em todas as variáveis, exceto flexão de quadril. Observou-se ainda, menor variação angular (flexão/extensão) no joelho do membro afetado quando comparado com o sadio.

Conclusões: $\mathrm{O}$ estudo indicou alterações na cinemática da marcha do membro acometido de indivíduos hemiparéticos após acidente vascular cerebral, comparando com a normalidade e com o membro contralateral, caracterizando a presença de um padrão flexor incomum. Essas alterações podem estar relacionadas com diminuição do equilíbrio e do controle motor, fraqueza muscular, déficit proprioceptivo, aumento do tônus, contraturas e deformidades.

DESCRITORES: acidente vascular cerebral; cinemática; fenômenos biomecânicos; marcha.

\section{ABSTRACT}

Aims: To evaluate linear and angular kinematics of gait in patients with spastic hemiparesis secondary to stroke considering reference values and the unaffected limb.

Methods: Cross-sectional study of adult patients with spastic hemiparesis secondary to stroke selected from the Physical Therapy Clinic of Universidade de Caxias do Sul (UCS). The kinematic gait analysis was performed at the Human Motion Biomechanical Analysis Laboratory at UCS according to Larouche's protocol. Descriptive statistics, one-sample $t$ test, and paired $t$ test were used for the data analysis. Statistical significance was set as $\mathrm{p}<0.05$.

Results: Eight adult male and female individuals aged 43 to 59 years were assessed. All variables indicated a significant difference in the linear kinematics of the gait of the affected limb. By comparing the lower limbs, stride length and rate were significantly higher in the affected hemibody. Angular kinematics was significantly different from reference values, except for hip flexion. There was a lower angular variation (flexion/extension) in the knee of the affected limb in comparison to the healthy one.

Conclusions: This study showed changes in the gait kinematics of the affected limb in hemiparetic patients after a stroke in comparison to normal patterns and to the contralateral limb, which indicates an unusual flexor pattern. These changes may be related to poor balance and motor control, muscle weakness, proprioceptive deficit, increased muscle tone, contractures, and deformities.

KEY WORDS: stroke; kinematics; biomechanical phenomena; gait. 
Abreviaturas: AVC, acidente vascular cerebral; IMC, índice de massa corporal; CLIFI, Clínica de Fisioterapia da Universidade de Caxias do Sul.

\section{INTRODUÇÃO}

A marcha é uma das funções mais utilizadas pelas pessoas; ela possibilita o deslocamento, a realização de atividades diárias, a manutenção da independência e da qualidade de vida. A eficiência da marcha depende da estabilidade no apoio, dos meios de progressão adequados e dos métodos para conservar energia. $\mathrm{O}$ sistema nervoso central é o principal responsável pela geração de ações motoras apropriadas, proporcionando uma estimativa da orientação dos segmentos corporais em relação um ao outro e ao meio [1].

Existem diversas alterações que afetam a marcha, entre elas a hemiparesia, que se refere à diminuição da força ou paralisia parcial afetando um hemicorpo. Sua causa pode estar relacionada a diferentes lesões encefálicas, entre elas o acidente vascular cerebral (AVC) [2]. Clinicamente, o AVC é caracterizado por início repentino, com sinais neurológicos focais ou globais, causados por isquemia ou hemorragia encefálica. A alteração funcional resultante, inclusive na motricidade, pode ser temporária ou permanente e parcial ou total, e a localização das sequelas depende da área do cérebro afetada $[3,4]$.

$\mathrm{Na}$ marcha hemiparética, também chamada de ceifante, observam-se diversas alterações biomecânicas e neuromusculares [5], devido à espasticidade no hemicorpo afetado $[2,6]$. Nesses indivíduos observamse também alterações na propriocepção, ausência de seletividade na ação de alguns grupos musculares e incapacidade para gerar dissociação entre a cintura escapular e a cintura pélvica. Por isso, tanto a fase de apoio quanto a de balanço estão prejudicadas, uma vez que ocorrerão mudanças nas dinâmicas do pé, joelho e quadril, que somadas à incoordenação e às alterações no controle postural, dificultam a progressão do membro para frente [7,8,9]. Destaca-se ainda que durante a fase de balanço o paciente faz uma inclinação da pelve para frente, por causa da fraqueza dos músculos abdominais. Também costuma fazer uma inclinação para o lado sadio, procurando melhor base de apoio e descarga eficiente de peso [10].

Entre as muitas formas de análise da marcha, destaca-se a realizada em laboratórios biomecânicos especializados, os quais fornecem dados tridimensionais e características específicas de cada indivíduo, possibilitando a adequada compreensão e descrição da marcha, o que beneficia os pacientes com diferentes comprometimentos $[1,11]$. A análise laboratorial é dividida em cinética e cinemática, sendo a cinemática subdividida em quantitativa linear e angular, o que possibilita descrever a marcha em características espaciais, temporais e espaciais/temporais [1,9].

A maioria dos pacientes com hemiparesia após AVC almeja ser capaz de andar novamente e de forma funcional. Atualmente, com a contribuição da tecnologia, os laboratórios biomecânicos são capazes de realizar uma análise de marcha muito eficiente, que analisa todas as variáveis desejadas e possibilita a identificação de alterações, fornecendo subsídios para os processos de reabilitação. Pensando na restauração da marcha com máxima eficiência, deve-se considerar a importância da avaliação cinemática da marcha e sua análise. Portanto, o objetivo deste estudo foi avaliar as alterações na cinemática angular e linear da marcha de pacientes hemiparéticos espásticos, comparando-as com a normalidade e com o hemicorpo sadio do mesmo indivíduo.

\section{MÉTODOS}

Este estudo de caráter observacional, comparativo, de abordagem transversal, foi aprovado pelo Comitê de Ética em Pesquisa da Universidade Federal de Ciências da Saúde de Porto Alegre sob no 362.784. A amostra foi do tipo intencional e não probabilística, composta por indivíduos de ambos os sexos, vinculados à Clínica de Fisioterapia da Universidade de Caxias do Sul (CLIFI). Foram considerados como critérios de inclusão pacientes adultos, com hemiparesia espástica após AVC, que deambulassem com ou sem dispositivo auxiliar, que já houvessem finalizado o tratamento fisioterapêutico ou estivessem em tratamento e em pré alta do serviço e que aceitassem participar do projeto assinando o termo de consentimento livre e esclarecido. Não foram incluídos indivíduos cadeirantes ou que apresentavam hemiparesia flácida, deficiência visual sem correção, alterações cardiovasculares sem acompanhamento, alterações cognitivas, mais de 60 anos de idade, índice de massa corporal (IMC) acima de $27 \mathrm{~kg} / \mathrm{m}^{2}$ ou dor aguda.

Após a liberação para acesso à CLIFI e aos prontuários, os possíveis participantes foram selecionados conforme os critérios de inclusão. Em seguida foi realizado contato com os pacientes, convidando-os a participar e explicando os procedimentos do estudo. Após a confirmação de participação, foi agendada a avaliação e os voluntários assinaram um termo de consentimento livre e esclarecido. 
As avaliações foram realizadas no laboratório de biomecânica de marcha localizado na Universidade de Caxias do Sul, que possui um sistema com protocolo para captação de dados cinemáticos e cinéticos. No presente estudo utilizou-se apenas o protocolo para captação de dados cinemáticos, o que é feito por meio de um sistema de cinemetria dotado de sete câmeras infravermelho integradas (VICON MX systems, Oxford Metrics Group, UK) que capturam a marcha de forma tridimensional, através dos marcadores reflexivos posicionados no corpo do indivíduo. Os dados cinemáticos foram processados em uma taxa de amostragem de $100 \mathrm{~Hz}$.

Os procedimentos para coleta de dados no laboratório foram baseados no protocolo de Laroche et al. [12]. Para adaptação dos participantes ao protocolo de avaliação, no local da coleta, primeiramente os sujeitos caminharam oito metros em linha reta, em velocidade auto selecionada. Para conseguirem realizar o contato com a plataforma, ora com o pé direito inteiro, ora com o pé esquerdo inteiro, os participantes eram orientados a manter o mesmo ritmo. Finalizada a familiarização com o processo de coleta, foram colocados os marcadores reflexivos nos seguintes pontos anatômicos dos indivíduos: crista ilíaca superior anterior direita e esquerda, crista ilíaca posterior direita e esquerda, coxa direita e esquerda, joelho direito e esquerdo, tíbia direita e esquerda, tornozelo direito e esquerdo, calcanhar direito e esquerdo e dedo do pé direito e esquerdo.

Durante a aplicação do protocolo de marcha o indivíduo realizava passos sobre as plataformas, sendo que em todas as tentativas o sujeito realizou o mesmo percurso treinado na familiarização. Tentativas foram realizadas até que oito passos fossem capturados integralmente [12]. Os pacientes usaram roupas leves, coladas ao corpo, que permitiram que fosse fácil a colocação dos eletrodos (sem movimentação) nos pontos anatômicos e lhes permitiram uma mobilidade adequada na deambulação.

As variáveis cinemáticas lineares analisadas foram: a) variáveis espaciais da marcha: comprimento do passo, largura do passo; b) variáveis temporais da marcha: cadência e tempo do passo; e c) variável espaço temporal: velocidade da marcha. Para as comparações das variáveis da cinemática linear dos indivíduos com a normalidade foram considerados os valores descritos por Neumann [1].

Para caracterização da amostra, os pacientes responderam a um questionário com perguntas sobre dados pessoais, história da doença atual e pregressa, informações sobre a lesão (como local, extensão e tempo), se usavam medicamentos e há quanto tempo realizavam fisioterapia. Após a resposta ao questionário, foram coletados os dados antropométricos dos pacientes, usando uma balança de chão (marca Sunrise, analógica) para o peso e uma fita métrica para a altura.

Os dados foram analisados através do programa SPSS Statistics 17.0. Para descrição das variáveis cinemáticas da marcha foi utilizada estatística descritiva com distribuição de frequência simples e relativa, bem como medidas de tendência central e de variabilidade. Para as comparações das variáveis cinemáticas da marcha do membro sadio com o afetado foi utilizado o teste $t$ pareado e para as comparações das mesmas variáveis com a normalidade foi utilizado o teste $\mathrm{t}$ para média de uma amostra, considerando como nível de significância um $p<0,05$ [13].

\section{RESULTADOS}

Foram avaliados oito participantes, com idades entre 43 e 59 anos (média 50,38 $\pm 6,67$ anos), todos com diagnóstico clínico de AVC, sendo cinco homens e três mulheres. O IMC variou entre 25,7 e 26,88 , o peso entre 58 e $93 \mathrm{~kg}$ (média $71,12 \pm 10,23 \mathrm{~kg}$ ) e a altura entre 1,50 a $1,86 \mathrm{~m}$ (média 1,65 $\pm 0,1 \mathrm{~m}$ ). O tempo de lesão variou de um a 17 meses (média 9,50 44,92 meses). Seis pacientes apresentavam o hemicorpo esquerdo acometido e dois o hemicorpo direito. Entre as medicações citadas, estavam antidepressivos e anti-hipertensivos.

As médias das características lineares e angulares encontradas foram comparadas com os valores de normalidade segundo Neuman [1]. Dentro das variáveis espaciais, o comprimento do passo estava diminuído em relação à normalidade e a largura do passo estava aumentada, ambos de forma significativa. Nas variáveis temporais, a cadência estava diminuída de forma significativa, comparada com a normalidade, sendo que para a variável tempo do passo não existem valores de normalidade. A velocidade também encontrava-se diminuída de forma significativa quando comparada com a normalidade (Tabela $\mathbf{1}$ ).

Nas variáveis angulares, a média da angulação de flexão de quadril estava de acordo com a normalidade, porém a flexão e a extensão de joelho, assim como a extensão de quadril estavam abaixo da normalidade, todas de forma significativa, demonstrando menor variação angular. Assim, comparando as médias das variáveis lineares e angulares com a normalidade, a amostra evidenciou a realização de um passo curto, alargado, em baixa velocidade, com angulações diminuídas, exceto flexão de quadril (Tabela 1). 
Tabela 1. Média das variáveis lineares e angulares em amostra de oito pacientes hemiparéticos espásticos após acidente vascular cerebral, comparadas com a normalidade

Tabela 2. Comparação da média das variáveis lineares e angulares entre o hemicorpo acometido e o não acometido, em amostra de oito pacientes hemiparéticos espásticos após acidente vascular cerebral

\begin{tabular}{|c|c|c|c|}
\hline Variáveis cinemáticas & $\begin{array}{c}\text { Pacientes } \\
\text { (Média } \pm \text { DP) }\end{array}$ & Normalidade* & $\mathbf{p}$ \\
\hline \multicolumn{4}{|l|}{ Espaciais } \\
\hline Comprimento do passo $(\mathrm{m})$ & $0,29 \pm 0,11$ & 0,72 & $<0,001$ \\
\hline Largura do passo (m) & $0,22 \pm 0,04$ & $0,07-0,09$ & $<0,001$ \\
\hline \multicolumn{4}{|l|}{ Temporais } \\
\hline Cadência (passos/min) & $57,22 \pm 26,98$ & 110 & 0,001 \\
\hline Tempo do passo (s) & $1,28 \pm 0,62$ & $t$ & \\
\hline \multicolumn{4}{|l|}{ Espaço-temporal } \\
\hline Velocidade $(\mathrm{m} / \mathrm{s})$ & $0,31 \pm 0,23$ & 1,37 & $<0,01$ \\
\hline \multicolumn{4}{|l|}{ Angulares } \\
\hline Flexão de quadril (graus) & $30,26 \pm 13,59$ & $30^{\circ}$ & 0,958 \\
\hline Flexão de joelho (graus) & $43,44 \pm 6,02$ & $60^{\circ}$ & $<0,001$ \\
\hline Extensão de quadril (graus) & $2,45 \pm 8,66$ & $-10^{\circ}$ & 0,005 \\
\hline Extensão de joelho (graus) & $9,28 \pm 9,27$ & 0 o & 0,025 \\
\hline
\end{tabular}

DP, desvio padrão; m, metros; s, segundos; passos/min, passos por minuto; m/s, metros/segundo.

* segundo Neumann DA. Cinesiologia do aparelho musculoesquelético. 2ª ed. Rio de Janeiro: Elsevier; 2011. † não encontrados dados na literatura.

\begin{tabular}{lccc}
\hline Variáveis cinemáticas & \multicolumn{2}{c}{ Membro inferior } & \\
\cline { 2 - 3 } & $\begin{array}{c}\text { Afetado } \\
\text { (Média } \pm \text { DP) }\end{array}$ & $\begin{array}{c}\text { Não afetado } \\
\text { (Média } \pm \text { DP) }\end{array}$ & $\mathbf{p}$ \\
\hline $\begin{array}{l}\text { Espaciais } \\
\quad \text { Comprimento do passo }(\mathrm{m})\end{array}$ & $0,34 \pm 0,11$ & $0,24 \pm 0,15$ & 0,087 \\
$\quad$ Largura do passo (m) & $0,22 \pm 0,04$ & $0,23 \pm 0,04$ & 0,560 \\
\hline $\begin{array}{l}\text { Temporais } \\
\quad \text { Tempo do passo (s) }\end{array}$ & $1,70 \pm 0,93$ & $0,86 \pm 0,31$ & $\mathbf{0 , 0 1 1}$ \\
Angulares & & & \\
$\quad$ Flexão de quadril (graus) & $32,61 \pm 20,25$ & $27,95 \pm 14,46$ & 0,572 \\
$\quad$ Flexão de joelho (graus) & $30,52 \pm 7,75$ & $56,37 \pm 10,45$ & $\mathbf{0 , 0 0 1}$ \\
$\quad$ Extensão de quadril (graus) & $5,18 \pm 12,73$ & $-0,29 \pm 10,75$ & 0,366 \\
$\quad$ Extensão de joelho (graus) & $6,16 \pm 14,76$ & $12,40 \pm 5,81$ & 0,206 \\
\hline
\end{tabular}

DP, desvio padrão; m, metros; s, segundos; passos/min, passos por minuto; m/s, metros/segundo.
Na comparação entre as médias das variáveis lineares do hemicorpo acometido com as do não acometido, o estudo mostrou que nas variáveis espaciais não houve diferença significativa entre as médias dos dois hemicorpos, enquanto na variável temporal, o tempo do passo foi significativamente maior no hemicorpo acometido do que no hemicorpo não acometido. Nas variáveis angulares, apenas os ângulos de flexão de joelho mostraram diferença significativa entre o hemicorpo afetado e o sadio (Tabela 2).

\section{DISCUSSÃO}

O presente estudo avaliou e analisou a marcha de pacientes com diagnóstico clínico de AVC e hemiparesia espástica, os quais demonstraram padrão de movimentação predominantemente em flexão e diversas alterações nas variáveis cinemáticas.
Sabe-se que o padrão da marcha hemiparética varia de acordo com o local e extensão da lesão cerebral. Após o AVC, observam-se diversos déficits motores referentes à marcha, a qual ocorre de forma desequilibrada, descoordenada e arrítmica; além de assimétrica e com variação no padrão de movimento [14]. Destaca-se ainda, o elevado gasto de energia, devido a mecanismos compensatórios que interferem na deambulação com máxima eficiência dos pacientes hemiparéticos $[15,16]$. Estudos anteriores já mostraram que a marcha de indivíduos hemiparéticos, quando comparada com indivíduos saudáveis, demonstra muitas diferenças [5], destacando-se a diminuição da velocidade, da cadência e do comprimento do passo, como observado na presente pesquisa, onde ambas as variáveis demonstraram diferença significativa quando comparadas com a normalidade [15]. 
Em relação à velocidade da marcha, os hemiplégicos em geral demonstram valores reduzidos em ambos os membros inferiores, com maior acometimento no membro espástico, indo ao encontro com o observado no presente estudo, que também identificou significativa diminuição da velocidade da marcha quando comparada com a normalidade. Este é um achado relevante, uma vez que, a velocidade da marcha é um forte indicador de funcionalidade após o AVC, e que ao aumentar a velocidade, o padrão de marcha melhora, considerando variáveis como passada, cadência, tempo de apoio, simetria e ativação muscular [14-18]. Em pacientes que sofreram AVC e apresentam hipertonia espástica, quanto maior for a duração do ciclo da marcha, mais haverá diminuição do tamanho do passo, da velocidade e da cadência, que são causados pela instabilidade na transferência de peso do membro inferior afetado para o sadio. Além disso, quanto maiores forem as exigências sobre o membro afetado, maiores serão as disfunções observadas no membro hemiparético $[10,19,20]$.

Ao considerar a normalidade, o comprimento do passo demonstrou estar diminuído e a largura do passo aumentada. Romero et al. [21], ao avaliarem a marcha de 11 indivíduos após AVC, encontraram resultados semelhantes. Os autores explicam que as retrações musculares, alterações de tônus, desequilíbrios posturais e a falta de seletividade no movimento geram aumento da base de suporte, redução do comprimento do passo e da passada [21].

Barela et al. [22] em estudo com 18 indivíduos, reforçam que essas alterações na normalidade da marcha são geradas pela falta de força muscular e dificuldade de descarregar peso no hemicorpo afetado. Isso gera compensações entre os membros para não perder o equilíbrio e para poder dar o próximo passo, como por exemplo, colocar rapidamente o membro não acometido à frente, para evitar o desequilíbrio [22]. Explicação essa que corrobora com o que se observa neste estudo, ao demonstrar que o tamanho do passo é menor no membro não afetado, do que no afetado.

Apesar da literatura não trazer parâmetros de normalidade para o tempo do passo, no presente estudo verificou-se que há aumento do tempo do passo no membro afetado em relação ao não afetado, caracterizando um ciclo mais lento. Todas essas alterações citadas podem também, ser justificadas pela diminuição do equilíbrio, a qual faz com que os pacientes precisem aumentar a base de suporte para se manterem verticalizados. As diminuições do comprimento do passo, do tempo do passo, da velocidade e da cadência devem-se à diminuição do controle motor, interferindo na seletividade muscular. Além disso, fraqueza muscular, déficit proprioceptivo, aumento de tônus, contraturas e deformidades ocasionam diminuição da flexibilidade muscular e angulação das articulações [6,10,23,24].

Após o AVC ocorrem diversas alterações biomecânicas, especialmente no membro hemiparético, como redução da amplitude dos movimentos das articulações e anormalidades no controle e ativação muscular [5,14]. A literatura destaca diversas alterações angulares, como a redução da flexão de quadril no contato inicial e a diminuição da flexão de joelho e aumento no quadril durante a retirada do pé do solo. Essas alterações estão relacionadas geralmente com a velocidade da marcha e com o grau de alteração e comprometimento motor $[15,16,26]$.

Ao analisar as variáveis angulares, comparando com os valores de normalidade e com o membro não afetado, os resultados demonstraram características diferentes do esperado para a marcha hemiparética. Divergindo da literatura, no presente estudo a média da angulação de flexão de quadril está de acordo com a normalidade, representada por $30^{\circ}$ de flexão de quadril $[1,15]$. Embora a literatura ressalte que há uma diminuição de flexão de quadril e joelho em pacientes com sequelas de $\mathrm{AVC}$, isso não foi observado no presente estudo. Destaca-se que embora os pacientes tenham demonstrado maiores valores de flexão de quadril, apresentaram menor variação de movimento angular, de extensão para flexão e viceversa, indicando que estão caminhando em flexão de membros inferiores (quadril e joelho).

Sinergias obrigatórias anormais e altamente estereotipadas emergem com a espasticidade [27]. Sullivan e Schmitz explicam que embora seja mais frequente a característica extensora de membros inferiores, o oposto também pode ocorrer; havendo portanto, dois padrões distintos de sinergia anormal, uma sinergia em flexão e uma em extensão [27]. No padrão flexor, o centro de gravidade encontra-se bem mais à frente da pelve que o usual, podendo decorrer de um tronco rígido, de contraturas em flexão de joelho/ quadril, flexão de joelho causada por sóleo e quadríceps fracos, hipertonicidade dos flexores de quadril, reflexo de retirada flexora e dominância da sinergia flexora durante a recuperação de um AVC [27].

A sinergia em flexão, observada no presente estudo, é composta de flexão, abdução e rotação externa do quadril; flexão do joelho; dorsiflexão e inversão do tornozelo e dorsiflexão do dedo do pé [27]. A flexão persistente do joelho devida à espasticidade dos 
isquiostibiais limita a efetividade do balanço terminal e restringe o avanço da coxa no apoio. Já a espasticidade do flexor do quadril limita a progressão no apoio médio e terminal, enquanto a ação mantida do quadríceps inibe a preparação no pré-balanço para o avanço do membro [6].

Acredita-se que as características observadas no membro não afetado, amplitude diminuída e aceleração aumentada, decorram da diminuição da descarga de peso no membro afetado. De acordo com Neumann ${ }^{1}$, no membro afetado ocorre uma limitação da extensão do joelho (contratura de flexão de joelho) que resulta em uma perna funcionalmente menor, afetando a cinemática das fases de apoio e oscilação da perna. A fase de apoio da perna, sem a extensão do joelho, precisa assumir uma posição de genuflexão, envolvendo o quadril, o joelho e o tornozelo, enquanto a fase de oscilação normal da perna necessita maior flexão do joelho e, possivelmente, do quadril, para tirar os dedos do solo [1].

Ainda sobre o mesmo aspecto, em relação ao membro inferior, ao tentar manter a ortostase, quando o controle motor possui baixa ação motora e reduzido tônus postural, a pelve inclina-se para frente ou para trás, ou ainda, para baixo sobre o lado afetado. O resultado dessa posição e da diminuição do controle motor é a flexão do quadril e do joelho. Essa combinação flexora associada à incapacidade de sustentação de peso sobre o lado afetado, colocam o tornozelo em posição inadequada [1].

Embora com número amostral pequeno, os resultados desta pesquisa fornecem parâmetros importantes em relação à análise cinemática da marcha de indivíduos com sequelas de AVC, com hemiparesia espástica e padrão flexor. Foram detectadas diminuições no comprimento do passo, na cadência e na velocidade, além de uma base de apoio maior. Observou-se pouca variação angular de flexão para extensão e vice-versa, indicando marcha em posição de flexão de membros inferiores. $\mathrm{O}$ membro afetado mostrou-se diferente do sadio tanto nas variáveis angulares quanto lineares. Pesquisas como esta podem auxiliar no tratamento adequado da hemiparesia, minimizando as alterações para tornar a marcha mais funcional.

\section{NOTA}

Declaração de conflitos de interesse

Os autores declaram não haver conflitos de interesse relevantes ao conteúdo deste estudo.

\section{REFERÊNCIAS}

1. Neumann D. Cinesiologia do aparelho musculoesquelético, fundamentos para a reabilitação. 2a ed. Rio de Janeiro: Elsevier; 2011.

2. Iwabe C, Diz M, Barudy DP. Análise cinemática da marcha em indivíduos com Acidente Vascular Encefálico. Rev Neurocienc. 2008;16(4):292-6.

3. Lianza S. Medicina de reabilitação. Rio de Janeiro: Guanabara Koogan; 2007.

4. Stokes M. Neurologia para fisioterapeutas. São Paulo: Premier; 2000.

5. Rosa MC, Marques A, Demain S, Metcalf CD. Lower limb co-contraction during walking in subjects with stroke: A systematic review. J Electromyogr Kinesiol. 2014 Feb;24(1):1-10. http://dx.doi.org/10.1016/j.jelekin.2013.10.016

6. Perry J, Schoneberger B. Análise de marcha: marcha patológica. São Paulo: Manole; 2005.

7. Sousa AS, Tavares JM.Interlimb coordination during step-to-step transition and gait performance. J Mot Behav. 2015;47(6): 563-74. http://dx.doi.org/10.1080/00222895.2015.1023391

8. Morioka S, Miyaoto S, Abe M. Relatioship between the center of gravity point in spontaneous standing and the middle point calculated from the center of gravity shifting distance to the non-paralytic and paralytic sides in hemiplegics after stroke. J Phys Ther Sci. 2003;15(2):99-103. http://dx.doi.org/10.1589/jpts.15.99

9. Rose J, Gamble JG. Marcha humana. 2ª ed. São Paulo: Premier; 2007.

10. Ottoboni C, Fontes SV, Fukujima MM. Estudo comparativo entre a marcha normal e a de pacientes hemiparéticos por acidente vascular encefálico: aspectos biomecânicos. Rev Neurocienc. 2002;10(1):5-9.

11. Araújo AGN, Andrade LM, Barros RML. Sistema para analise cinemática da marcha humana baseado em videogrametria. Fisioter Pesqui. 2004;1(1):3-10.

12. Laroche D, Duval A, Morisset C, Beis JN, D'athis P, Maillefert JF, Ornetti P. Test-retest reliability of 3D kinematic gait variables in hip osteoarthritis patients. Osteoarthritis Cartilage. 2011 Feb;19(2):194-9. http://dx.doi.org/10.1016/j.joca.2010.10.024

13. Callegari-Jacques SM. Bioestatística: princípios e aplicações. Porto Alegre: Artmed; 2003.

14. Wist S, Clivaz J, Sattelmayer M. Muscle strengthening for hemiparesis after stroke: A meta-analysis. Ann Phys Rehabil Med. 2016 Apr;59(2):114-24. http://dx.doi.org/10.1016/j.rehab.2016.02.001 
15. Polese JC, Nascimento LR, Faria CDCM, Laurentino GEC, Rodrigues de Paula F, Ada L, Teixeira SLF. Percepção de hemiplégicos crônicos sobre o uso de dispositivos auxiliares na marcha. Rev Panam Salud Publica. 2011;30(3):204-8. http://dx.doi.org/10.1590/ S1020-49892011000900003

16. Stowe S, Hopesb J, Mulley G. Gerotechnology series: 2. Walking aids. Eur Geriatr Med. 2010;1(2):122-7. http://dx.doi.org/10.1016/j. eurger.2010.02.003

17. Kerrigan DC, Karvosky ME, Riley PO. Spastic paretic stiff-legged gait: joint kinetics. Am J Phys Med Rehabil. 2001 Apr;80(4): 244-9. http://dx.doi.org/10.1097/00002060-200104000-00002

18. Burnfield JM, Buster TW, Goldman AJ, Corbridge LM, Harper-Hanigan K. Partial body weight support treadmill training speed influences paretic and non-paretic leg muscle activation, stride characteristics, and ratings of perceived exertion during acute stroke rehabilitation. Hum Mov Sci. 2016 Feb 1;47:16-28. http://dx.doi.org/10.1016/j.humov.2016.01.012

19. Corrêa FI, Soares F, Andrade DV, Gondo RM, Reres JÁ, Fernandes AO. Atividade muscular durante a marcha após acidente vascular encefálico. Arq Neuropsiquiatr. 2005;63(3b):847-51. http://dx.doi.org/10.1590/S0004-282X2005000500024

20. Silva A, Sousa AS, Silva C, Tavares JM, Santos R, Sousa F. Ankle antagonist coactivation in the double-support phase of walking: Stroke vs. healthy subjects. Somatosens Mot Res. 2015;32(3):153-7. http://dx.doi.org/10.3109/08990220.2015.1012492

21. Romero VM, Carvalho PTC, Laraia SEM, Soares NC. Análise das variáveis de distância da marcha de pacientes com acidente vascular encefálico. Conscientiae Saúde. 2008;7(3):329-34.

22. Barela J, Whitall J, Black P, Clark J. An examination of constraints affecting the intralimb coordination of hemiparetic gait. Hum Mov Sci. 2000 July;19(2):251-73. http://dx.doi.org/10.1016/S0167-9457(00)00014-2

23. Silva LLM, Moura CEM, Godoy JRP. A marcha no paciente hemiparético. Universitas. 2005;3(2):261-73.

24. Martins FLM, Guimarães LHCT, Vitorino DFM, Souza LCF. Eficácia da eletroestimulação funcional na amplitude de movimento de dorsiflexão de hemiparéticos. Rev Neurocienc. 2004;12(2):103-9.

25. Buurke JH, Hermens HJ, Erren-Wolters CV, Nene AV. The effect of walking aids on muscle activation patterns during walking in stroke patients. Gait Posture. 2005 Oct;22(2):164-70. http://dx.doi.org/10.1016/j.gaitpost.2004.09.003

26. Hesse S, Werner C, Paul T, Bardeleben A, Chaler J. Influence of walking speed on lower limb muscle activity and energy consumption during treadmill walking of hemiparetic patients. Arch Phys Med Rehabil. 2001 Nov;82(11):1547-50. http://dx.doi. org/10.1053/apmr.2001.26607

27. O’Sullivan, SB, Schimitz TJ. Fisioterapia: avaliação e tratamento. 4a ed. São Paulo: Manole; 2004. 